\title{
Use of anion-aromatic interactions to position the general base in the ketosteroid isomerase active site
}

\author{
Jason P. Schwans ${ }^{a}$, Fanny Sundena, Jonathan K. Lassila ${ }^{a}$, Ana Gonzalez ${ }^{b}$, Yingssu Tsai ${ }^{b, c}$, and Daniel Herschlag ${ }^{a, c, 1}$ \\ Departments of ${ }^{\mathrm{a} B i o c h e m i s t r y}$ and ${ }^{\mathrm{C} C h e m i s t r y, ~ S t a n f o r d ~ U n i v e r s i t y, ~ S t a n f o r d, ~ C A ~ 94305 ; ~ a n d ~}{ }^{\mathrm{b}}$ Stanford Synchrotron Radiation Lightsource, Stanford Linear \\ Accelerator Center National Accelerator Laboratory, Menlo Park, CA 94025
}

Edited* by JoAnne Stubbe, Massachusetts Institute of Technology, Cambridge, MA, and approved May 29, 2013 (received for review April 20, 2012)

\begin{abstract}
Although the cation-pi pair, formed between a side chain or substrate cation and the negative electrostatic potential of a pi system on the face of an aromatic ring, has been widely discussed and has been shown to be important in protein structure and protein-ligand interactions, there has been little discussion of the potential structural and functional importance in proteins of the related anion-aromatic pair (i.e., interaction of a negatively charged group with the positive electrostatic potential on the ring edge of an aromatic group). We posited, based on prior structural information, that anion-aromatic interactions between the anionic Asp general base and Phe54 and Phe116 might be used instead of a hydrogen-bond network to position the general base in the active site of ketosteroid isomerase from Comamonas testosteroni as there are no neighboring hydrogenbonding groups. We have tested the role of the Phe residues using site-directed mutagenesis, double-mutant cycles, and high-resolution $X$-ray crystallography. These results indicate a catalytic role of these Phe residues. Extensive analysis of the Protein Data Bank provides strong support for a catalytic role of these and other Phe residues in providing anion-aromatic interactions that position anionic general bases within enzyme active sites. Our results further reveal a potential selective advantage of Phe in certain situations, relative to more traditional hydrogen-bonding groups, because it can simultaneously aid in the binding of hydrophobic substrates and positioning of a neighboring general base.
\end{abstract}

enzyme catalysis | general-base catalysis | noncovalent interactions

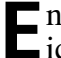
nzymes use the same functional groups to achieve "chemical catalysis" as small-molecule catalysts, and yet enzymes attain much greater rate enhancements. A distinguishing feature of enzymes is that their reactions occur in highly specialized active sites that use noncovalent interactions to precisely position enzymatic functional groups relative to substrates and relative to other active-site features. Understanding how these groups are positioned within enzyme active sites is key for understanding the differences between enzymes and small-molecule catalysts.

It is generally recognized that hydrophobic interactions can help bind and position substrates in enzyme active sites $(1,2)$. Beyond hydrophobic interactions, much attention has focused on the importance of hydrogen bonds in precisely positioning active-site groups directly involved in the chemical reaction (e.g., the catalytic triad in serine proteases), and the importance of hydrogen-bonding groups is supported by mutagenesis in many cases (e.g., refs. 1 and 3-5).

In addition to hydrogen bonds, the cation-pi pair, formed between a side-chain or substrate cation and the negative electrostatic potential associated with the face of a pi system, has been widely discussed in hundreds of literature reports and has been shown to be important in both protein structure and proteinligand interactions (e.g., refs. 6 and 7). There has been much less discussion, however, of the potential importance of the related anion-aromatic pair in protein structure and function (i.e., interaction of a negatively charged side chain and the positive electrostatic potential associated with the ring edge of an aromatic group; Fig. 1). ${ }^{\dagger}$ Several decades ago, Thomas et al. analyzed 28 protein structures and reported a preference for oxygen atoms near the ring edge of phenylalanine residues (13). The authors suggested that the partial positive charge at the edge of the aromatic ring favors positioning oxygen atoms around the ring edge (13). Howell and coworkers recently carried out a much larger scale analysis and showed that the anionic oxygen atoms of Asp and Glu carboxylate groups are commonly situated near the ring edge of the aromatic residues in protein structures $(8,9)$. These results and prior computational studies in small molecule systems have suggested that anion-aromatic pairs play important roles in protein stability and ligand binding, with energies similar to those of cation-pi interactions $(8,9)$. The results presented herein provide evidence for a role for anion-aromatic interactions in catalysis.

In bacterial ketosteroid isomerase (KSI) from Comamonas testosteroni (tKSI), an active-site aspartate general base (Asp38) deprotonates the substrate, generating a dienolate intermediate, and reprotonates the intermediate to form the product (Fig. 2A). In contrast to the many examples of Asp and Glu general bases positioned within active sites by hydrogen-bond networks (14), inspection of numerous tKSI structures reveals no hydrogenbonding groups adjacent to the Asp general base. Rather, the ring edge of a Phe residue, Phe54 for one oxygen and Phe116 for the other oxygen, is situated next to these anionic oxygen atoms (Fig. $2 B)(15,16)$. We posited, based on this structural information, that anion-aromatic interactions between the anionic Asp and Phe54 and Phe116 were used instead of a hydrogen-bond network to position the general base in the KSI active site and facilitate catalysis. Our results, using site-directed mutagenesis, doublemutant cycles, high-resolution X-ray crystallography, and analysis of a large number of enzyme structures in the Protein Data Bank (PDB) support this proposal and suggest a potential functional advantage and evolutionary pathway for introducing Phe residues that can aid in both the binding of a hydrophobic substrate and the positioning of a nearby general base.

\section{Results and Discussion}

We first describe functional and structural results that suggest Phe54 and Phe116 position the anionic general base in the tKSI active site, consistent with anion-aromatic interactions. We next test this model via statistical analysis of available X-ray structures. Finally, we describe results that suggest an additional role for

Author contributions: J.P.S., F.S., J.K.L., A.G., Y.T., and D.H. designed research; J.P.S., F.S J.K.L., A.G., and Y.T. performed research; J.P.S., F.S., J.K.L., A.G., Y.T., and D.H. analyzed data; and J.P.S., F.S., J.K.L., and D.H. wrote the paper.

The authors declare no conflict of interest.

*This Direct Submission article had a prearranged editor

Data deposition: The atomic coordinates and structure factors have been deposited in the Protein Data Bank, www.pdb.org (PDB ID codes 3UNL and 4L7K).

${ }^{1}$ To whom correspondence should be addressed. E-mail: herschla@stanford.edu.

This article contains supporting information online at www.pnas.org/lookup/suppl/doi:10. 1073/pnas.1206710110/-/DCSupplemental.

${ }^{\dagger}$ The term anion-pi was used in a recent computational study to describe this interaction $(8,9)$. We use the term anion-aromatic for this interaction because: $(i)$ the interaction involves the partially positive aromatic ring edge, not the face of the aromatic system, where the pi molecular orbitals are located; and (ii) the term anion-aromatic distinguishes this little-discussed interaction from the widely discussed anion-pi pair [interaction of a negatively charged group with the face of an electron deficient aromatic system (e.g., refs. 10-12)]. 


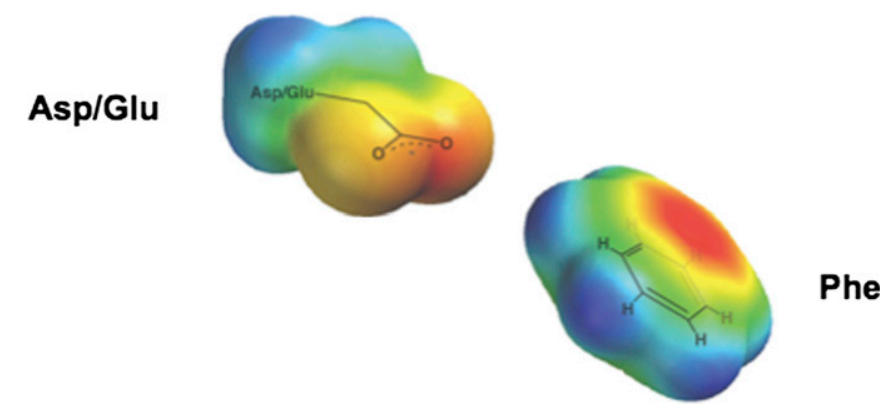

Fig. 1. Schematic of the anion-aromatic interaction. The negatively charged side chain is situated adjacent to the positive electrostatic potential associated with the ring edge of an aromatic group.

Phe116 in remote substrate binding interactions and how this second role can provide an evolutionary advantage and potential evolutionary pathway for the use of Phe in general-base positioning.

Effects of Phe54 and Phe116 Mutations on KSI Activity. We first determined $k_{\text {cat }}$ and $K_{\mathrm{M}}$ for the Phe54Ala and Phe116Ala mutants using the substrate 5(10)-estrene-3,17-dione [5(10)EST]. We used $5(10)$ EST to ensure that a chemical step is rate-limiting in all cases and that $K_{\mathrm{M}}$ reflects binding (17). The Phe54Ala and Phe116Ala mutations decreased $k_{\text {cat }}$ by 33 - and 24 -fold, respectively (Fig. $3 A$ ). Effects on $K_{\mathrm{M}}$ (Fig. $3 B$ ) are discussed below.

The rate decrease for the Phe54Ala mutation is similar to the 10 -fold rate reduction that Choi and coworkers reported previously for Phe56Ala in the related Pseudomonas putida KSI (pKSI) for reaction of this substrate (18). In pKSI, Trp120 replaces Phe116, and the Trp120Ala mutation led to a 68-fold rate decrease (18).

If Phe54 and Phe116 provide simple hydrophobic interactions to position the general base, then bulky hydrophobic residues might be able to substitute for Phe at these positions in tKSI. To probe this possibility, we mutated Phe54 and Phe116 to Val and Ile. These mutations led to the same rate decreases as for the Ala mutation for Phe54 and only two- to threefold smaller decreases for Phe116 (Fig. 3A). Mutation of Phe116 to Ala, Val, and Ile also led to similar $\sim$ sevenfold increases in $K_{\mathrm{M}}$ (Fig. $3 B$ ), suggesting a role for the aromatic Phe side chain in substrate-binding interactions, as described below.

X-Ray Structure of Phe54Gly. To directly evaluate whether ablation of a neighboring Phe residue affects the position of the general base, we turned to structure. We were unable to obtain diffracting crystals of the Phe54Ala and Phe116Ala mutants but were able to solve the crystal structure of tKSI Phe54Gly (PDB ID code 3UNL). Mutation of Phe 54 to Gly led to a 28 -fold decrease in $k_{\text {cat }}$, the same effect with error as the 33-fold rate decrease observed for mutation to Ala (Table S1). The overall Phe54Gly structure obtained at 2.5 - $\AA$ resolution was superimposable with that of previously determined wild-type tKSI (PDB ID code 8CHO; $2.3 \AA$ ) (16), with a root-mean-square deviation of $0.4 \AA$ for the backbone atoms (Fig. 4). The electron-density map shows well-defined densities for the modeled atomic positions of Asp38 in each of the chains in the asymmetric unit, with no indication of alternative side-chain orientations (Fig. S1). Whereas the Asp38 carboxylate is superimposable with wild-type KSI in one of the four chains in the asymmetric unit, the Asp38 carboxylate is displaced $0.8 \AA$ relative to wild-type KSI in the remaining three chains, suggesting that mutation of a neighboring Phe residue can affect the general-base positioning. Additional functional and structural tests described below support this interpretation of the KSI X-ray crystallographic results: a role of the Phe residue in general-base positioning.

Double-Mutant Cycles to Test the Role of Phe54 and Phe116 in Positioning the General Base in the KSI Active Site. If Phe54 and Phe116 were important for positioning the general base in the KSI active site, then we would expect mutation of these residues to have smaller deleterious effects in KSI with an already mispositioned general base (Fig. 5). Alternatively, if the rate effects from the Phe mutations were not related to positioning of the general base, then mutation of Phe54 and Phe116 would be expected, in the simplest scenario, to have the same deleterious effect in KSI with a positioned and mispositioned general base (Fig. 5). These comparisons delineate a double-mutant cycle, depicted in Fig. 5, that can provide an incisive test of the potential functional connection between the active-site Phe residues and positioning of the general base.

Prior studies have used mutations that add or remove a sidechain methylene group to test the importance of positioning active-site carboxylate groups (19-24). Such mutations are typically highly deleterious and have been shown to displace the position of the carboxylate by $\sim 1 \AA$ relative to its wild-type position in several cases (19-23), a displacement similar to that observed for the Phe54Gly mutation described above.

To directly test whether the Asp38Glu mutation perturbs the position of the general base in the KSI active site, we determined the crystal structure of tKSI Asp38Glu (PDB ID code 4L7K). The overall Asp38Glu structure, determined at 2.1-Å resolution, was superimposable with that of previously determined wild-type tKSI (PDB ID code 8CHO; $2.3 \AA$ ) (16), with a root-mean-square deviation of $0.4 \AA$ for the backbone atoms (Fig. S2). Whereas the refined positions of active-site residues surrounding residue 38 were superimposable with wild-type KSI, the Glu carboxylate is displaced $\sim 1.5 \AA$ from the position of the wild-type Asp carboxylate in all 12 chains of the asymmetric unit (Fig. S2; $1.5 \pm 0.2$ $\AA$ for the 12 active sites in the mutant unit cell). Thus, the Asp38Glu mutation appears to misposition the general base relative to wild-type KSI without introducing additional activesite structural rearrangements, as did mutation of Phe54 (Fig. 4).

To assess the interplay of the positioned general base with the role of the adjacent Phe residues, we compared the functional consequences of mutating Phe54 and Phe116 with Asp38, a positioned general base, versus the consequences with Asp38Glu, a mispositioned general base, via the double-mutant cycle outlined in Fig. 5. The Asp38Glu mutation led to a decrease of 240fold for $k_{\text {cat }}$ (Fig. $6 A$ ), similar to the 300 -fold decrease reported previously with a different KSI substrate (5-androstene-3,17-dione) (20). Whereas the Phe54Ala and Phe116Ala mutants reduce $k_{\text {cat }}$

Fig. 2. Potential anion-aromatic interactions position the general base in KSI. (A) Mechanism of KSIcatalyzed isomerization. (B) Space-filling representation of wild-type unbound tKSI shows Asp38 positioned near the edges of Phe54 and Phe116 (PDB ID code $8 \mathrm{CHO}$ ) with angles of interaction of $17.8^{\circ}$ and $2.6^{\circ}$, respectively. The angle is defined as that between the Asp carboxylate oxygen atom, the center point of the Phe ring, and the plane of the

A

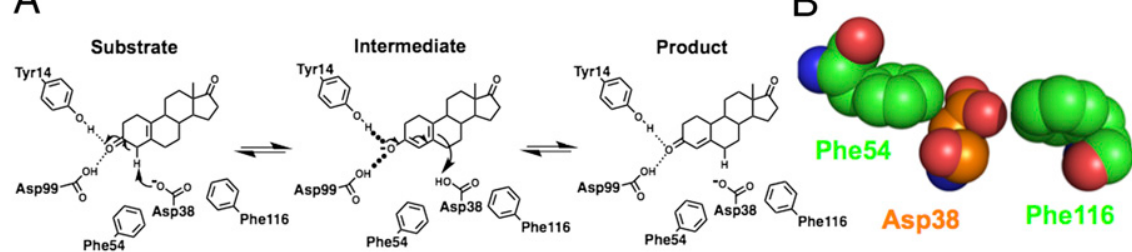

Phe ring. An angle of $0^{\circ}$ would correspond to the carboxylate positioned in the plane of the Phe ring, and an angle of $90^{\circ}$ would correspond to the carboxylate positioned above the face of the Phe ring (Fig. 7). 
A

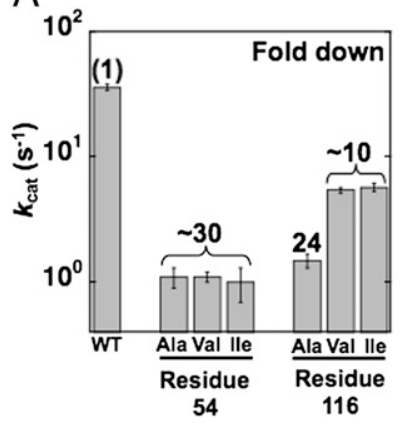

B

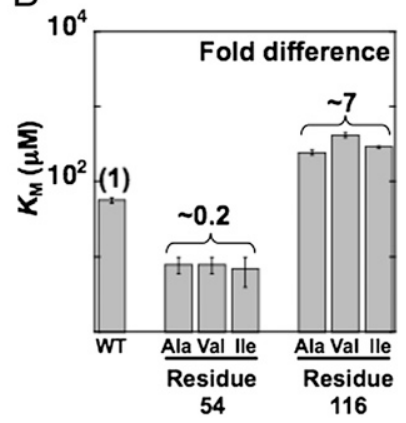

Fig. 3. Effects of Phe54 and Phe 116 mutations on $k_{\text {cat }}(A)$ and $K_{\mathrm{M}}(B)$. Values and errors are averages and SDs from three or more independent measurements and are from Table S1.

by $\sim 30$-fold when introduced in wild-type KSI, as noted above, these same mutations had no effect when made in an enzymatic background with Asp38 mutated to Glu (Fig. 6A). In other words, the Phe mutations only had deleterious effects on $k_{\text {cat }}$ when Asp was present. These results indicate a functional interaction between Phe54 and Phe116 and the general base.

Although the role of the Phe residues in positioning is supported by the double-mutant cycle and the inability of hydrophobic residues to replace Phe is consistent with an important anion-aromatic interaction, it is possible that a high geometric stringency prevents functional substitution of Phe by other hydrophobic residues. Thus, additional data are needed to distinguish the model of electrostatic interactions with the Phe positive edge positioning the general base from the alternative model of only spatial effects being important for positioning the general base. To evaluate these possibilities, we turned to the protein structural database.

Using the X-Ray Structural Database to Test the Hypothesis That Anion-Aromatic Interactions Position Carboxylate General Bases. If only spatial and not electrostatic interactions with the positive edge of Phe are important for positioning a carboxylate general base, then there should be no preference for Asp and Glu general bases to be situated near the edge relative to the rest of the Phe surface. Prior work by Howell and coworkers demonstrated through both quantum mechanical calculations and statistical analysis of structures in the PDB that positioning negatively charged residues near the positive edge of aromatic residues is energetically favorable $(8,9)$. Here, we extend this approach to evaluate whether Asp and Glu general bases are frequently positioned near the positive edge of aromatic residues, recognizing that the frequency of observation of a molecular state is related to free energy (e.g., refs. 25-27).

We first used the Catalytic Site Atlas (28) and PDB (http://rcsb. org/pdb) to identify active-site Asp and Glu residues that had nearby Phe residues (side-chain carboxylate oxygen atoms within $6 \AA$ of the center of a Phe ring). We then used the Catalytic Site Atlas (28, 29), MACiE (Mechanism, Annotation, and Classification in Enzymes) (30), BRaunschweig ENzyme DAtabase (BRENDA) (31), and the primary literature to determine whether the active-site carboxylate residues with the nearby Phe residues have been implicated as general bases. We identified 246 nonredundant enzymes with Asp or Glu residues implicated as general bases and with a nearby Phe residue. The angle between the Asp or Glu residue and the Phe ring was defined by the closest carboxylate oxygen atom, the center point of the Phe ring, and the plane of the Phe ring (Fig. $7 A$ ). To account for the differences in available $3 \mathrm{D}$ space for side-chain interactions near the ring edge relative to the rest of the Phe surface, we determined the expected distribution of angles for Asp or Glu residues relative to Phe based

on available volume (Experimental Methods and Table S3). A histogram of the analysis reveals that catalytic Asp and Glu residues show a preference for an edgewise interaction of the Phe ring with the carboxylate groups that is substantially greater than the distribution expected from available volume (Fig. 7B). These observations for carboxylates implicated as general bases mirror previous conclusions for structural anion-aromatic interactions $(8$, 9) and provide strong support for a frequent role of anion-aromatic interactions in the positioning of anionic general bases.

We next evaluated whether aromatics, and not alternative hydrophobic groups, are conserved neighbors of these general bases. Sequence similarity searches were carried out using the UniProt Knowledgebase for enzymes in Table S4 with an interaction angle of $0-10^{\circ}$ (32). To control for potential bias from the starting sequences, additional searches were performed starting with sequences that did not contain a Phe neighboring the general base. We found that for 67 of 82 enzymes evaluated, $>90 \%$ of the sequences contained an aromatic residue at the position of the neighboring Phe residue (Table $\mathrm{S} 5 A$ ). For KSI, an aromatic residue at position 54 was found in $98 \%$ of the sequences, respectively, with Phe most highly represented. At position 116, Trp is the most highly represented residue, and $97 \%$ of the sequences have an aromatic at this position (Table S5B). Structures of KSI from $P$. Putida show the Trp indole nitrogen situated to form a hydrogen bond with the Asp general base (33). The substitution of Phe at the position of a Trp hydrogen-bond donor is consistent with the model that an anion-aromatic interaction can substitute for a hydrogen-bonding residue. Although substitution of active-site residues are expected to occur less frequently than surface residues because there are multiple constraints in and around active sites, these results provide strong support for a role for aromatic residues, rather than generic hydrophobic groups, in interactions with carboxylate general bases.

We performed an additional analysis to determine whether aromatic residues commonly reside near those Asp and Glu general bases that do not have nearby hydrogen-bonding groups to position the carboxylate group. We found that aromatic residues were highly represented near carboxylate general bases without nearby hydrogen-bonding groups and that Phe residues, in particular, were common. Indeed, for 46 of 72 Asp or Glu general bases that we identified with no hydrogen bond within $3.2 \AA$, an aromatic residue was present within $4.0 \AA$. The Asp or Glu general bases in the remaining 26 structures had hydrogen bonds to crystallographically identified water molecules (20 structures), were clearly mispositioned (5 structures), or had low electron density (1 structure; see SI Text and Table S6 for details). These observations

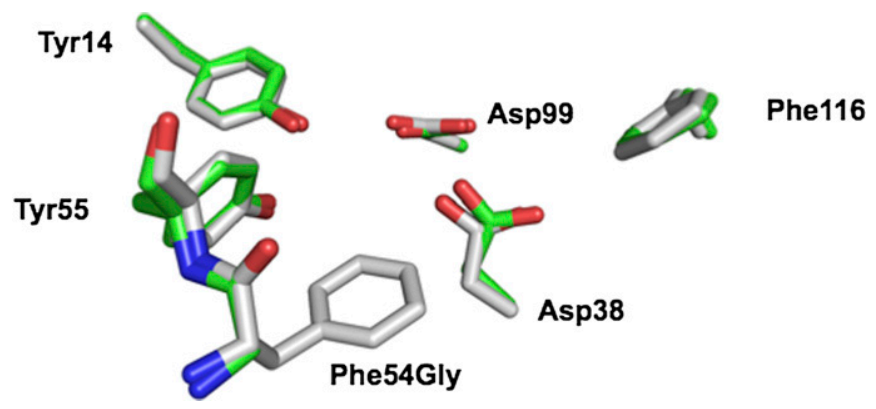

Fig. 4. Crystal structure of tKSI Phe54Gly shows the position of an Asp38 carboxylate oxygen atom is displaced $\sim 1.0 \AA$ relative to wild-type tKSI in three of the four chains in the asymmetric unit. Superposition of the tKSI Phe54Gly structure determined herein (PDB ID code 3UNL; carbon atoms are colored green) and the previously determined $2.3 \AA$ wild-type tKSI structure (PDB ID code 8CHO; carbon atoms are colored white). Only one chain from the asymmetric unit is shown for clarity. The overall root-mean-square deviation between the two structures for backbone atoms is $0.4 \AA$. X-ray data and refinement statistic are listed in Table S2. 

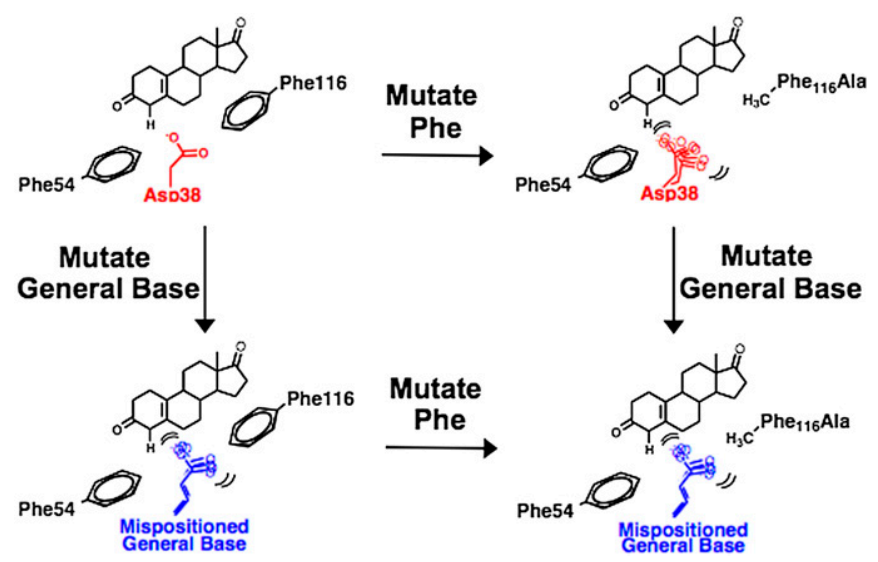

Fig. 5. Double-mutant cycle to test the role of Phe54 and Phe116 positioning the general base in KSI. According to the model presented in the text, Phe54 and Phe116 position the Asp38 general base in the active site of wild-type KSI. Mutation of Asp38 to a hypothetical "mispositioned" general base allows the general base to access a dominant alternate conformation (vertical arrows). Mutation of Phe116 or Phe54 affects the positioning of the general base starting from an enzyme with a positioned general base (top horizontal arrow) but does not have an effect for an enzyme with a mis positioned general base (bottom horizontal arrow).

support the model that interactions with the positive edge of aromatic residues are important for positioning carboxylate general bases in enzyme active sites.

Binding Effects and an Evolutionary Pathway for Using Anion-Aromatic Interactions to Position the General Base in KSI. Mutation of Phe54 and Phe116, which facilitate catalysis $\left(k_{\text {cat }}\right)$, also led to increased and decreased binding, respectively. We now turn to further investigation of these binding effects. Our results provide an intriguing model for why tKSI and certain other enzymes may be more likely to use anion-aromatic interactions in generalbase positioning.

Effects of Phe54 Mutations on $\boldsymbol{K}_{\mathbf{M}}$. Mutation of Phe54 to Ala, Gly, $\mathrm{Val}$, and Ile led to a $\sim$ fivefold decrease in $K_{\mathrm{M}}$ relative to wildtype KSI (Fig. $3 B$ and Table S1). The lower $K_{\mathrm{M}}$ values, coupled with the displacement of Asp38 in the Phe54 mutant (Fig. 4), suggest that substrate binding is increased in KSI with a mispositioned general base relative to a positioned general base in wild-type KSI. Close juxtaposition of the anionic general base near the hydrophobic steroid substrate in the Michaelis complex for wild-type KSI appears to be destabilizing relative to an enzyme with a mispositioned general base that can access alternative conformations that reduce unfavorable interactions between the general base and steroid substrate (Fig. S3 and ref. 34). Thus, perturbations that allow rearrangement of the Asp general base and, thereby, reduce these unfavorable ground-state interactions are predicted to increase substrate binding, as observed for the Phe54 mutations (Fig. $3 B$ and Table S1).

Testing the Role of Phe116 in Substrate-Binding Interactions. In the simplest scenario, mutations of Phe116 would also increase substrate binding. However, $K_{\mathrm{M}}$ increased $\sim 10$-fold upon mutation of Phe116 to Ala, Val, and Ile, which corresponds to decreased overall binding (Fig. $3 B$ ). The crystal structure of tKSI Asp38Asn bound to equilenin, a transition state analog, shows that Phe116 is situated near the B-D rings of the steroid (Fig. $8 A$ ), so that Phe116 could contribute binding interactions with the distal rings. In contrast, Phe54 is not situated near the distal steroid rings. To test the possible contribution of Phe116, we compared KSI catalysis of reactions of a single-ring substrate lacking the B-D rings (3-cyclohexen-1-one; $\mathrm{S}_{\text {mini }}$ ) and a multiple- ring steroid [5(10)EST; $\mathrm{S}_{\text {full }}$; Fig. $8 B$ ]. If remote binding interactions with Phe116 contribute to catalysis, then a simple expectation would be that mutation of Phe116 would not increase $K_{\mathrm{M}}$ for reaction with the single-ring substrate.

The $k_{\text {cat }}$ value of $17 \mathrm{~s}^{-1}$ for reaction of $\mathrm{S}_{\text {mini }}$ with wild-type tKSI is within twofold of the $k_{\text {cat }}$ value for reaction with $\mathrm{S}_{\text {full }}$, suggesting that binding interactions with the distal steroid rings are not important for specific transition-state stabilization, as previously observed for wild-type pKSI (35). Similar to the previously reported $\sim 10^{4}$-fold difference in $K_{\mathrm{M}}$ for reaction of $\mathrm{S}_{\text {full }}$ and $\mathrm{S}_{\text {mini }}$ with wild-type pKSI (35), the $K_{\mathrm{M}}$ value for reaction with $S_{\text {mini }}$ is $\sim 5,000$-fold higher than for reaction with $S_{\text {full }}$ for wild-type tKSI, suggesting that remote binding interactions are important for tKSI catalysis of reaction of free $\mathrm{S}$ (Fig. 8C). However, in contrast to the $\sim 10$-fold increase in $K_{\mathrm{M}}$ observed with $\mathrm{S}_{\text {full }}$ for the Phe116Ala mutation, the $K_{\mathrm{M}}$ value for $\mathrm{S}_{\text {mini }}$ was unaffected by the Phe116Ala mutation (Fig. $8 D$ ) and $k_{\text {cat }} / K_{\mathrm{M}}$ was correspondingly less affected for $\mathrm{S}_{\text {mini }}$ than $\mathrm{S}_{\text {full }}$ (Fig. $8 E$ ). Our results strongly suggest that Phe116 contributes to KSI catalysis by remote substrate-binding interactions, so that Phe116 exhibits a dual role in substrate binding and in general-base positioning.

Possible Pathways for the Introduction of Anion-Aromatic Interactions. Carter and Wells addressed the question of how the catalytic triad of a serine protease could have evolved, given that it acts cooperatively $(3,4)$. They showed that a particular pathway of addition gave a progressive increase in activity, whereas others did not $(3,4)$. The Phe54Ala/Phe116Ala double mutant led to the same decrease in $k_{\text {cat }}$, within twofold, as the individual Phe mutations (Table S1), indicating that the mutations are functionally cooperative and suggesting that the presence of a single Phe is insufficient to provide significant positioning of Asp38. How could such cooperative functional groups arise through evolution?

The introduction of Phe54 alone to the double mutant (to give the Phe116Ala mutant) gives no significant increase in catalytic power (Fig. 9 and Table S1), suggesting that this would not be a viable pathway in our simplified evolutionary analysis. Introduction of Phe116 alone (to give the Phe54Ala mutant) gives no enhancement of catalysis with bound substrate (Fig. 9 and Table S1) but does decrease $K_{\mathrm{M}}$ and, thereby, increases $k_{\text {cat }} / K_{\mathrm{M}}$. As described above, this effect arises from a binding interaction with the steroid B-D rings (Fig. 8), not from enhancement of general-base catalysis. However, once Phe116 is in place, introduction of the second Phe at position 54 provides positioning of the Asp general base, via interactions with both Phe residues,

A
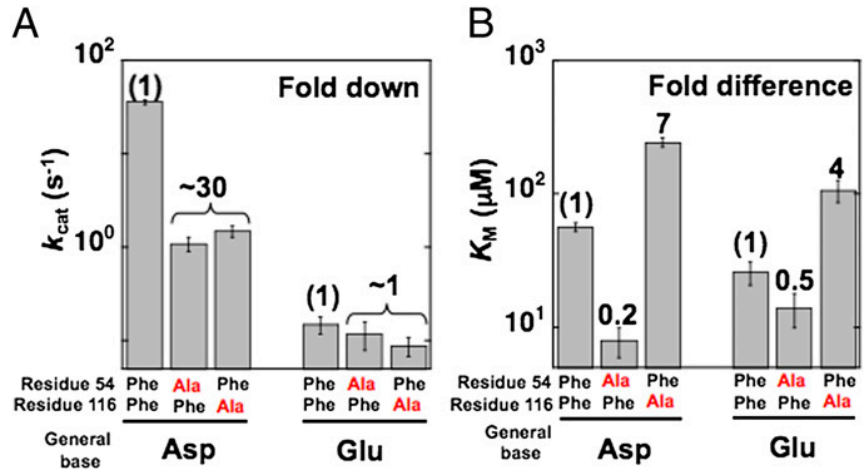

Fig. 6. Double-mutant cycle analysis to test the role of Phe54 and Phe116 positioning the general base in the $\mathrm{KSI}$ active site. Effects on $k_{\text {cat }}(A)$ and $K_{\mathrm{M}}$ (B) for mutation of Phe54 and Phe116 in KSI with a positioned Asp general base and a mispositioned Asp38Glu general base. Values and errors are averages and SDs from three or more independent measurements and are from Fig. 3 and Table S1. 


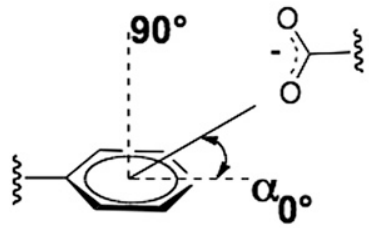

\section{B}

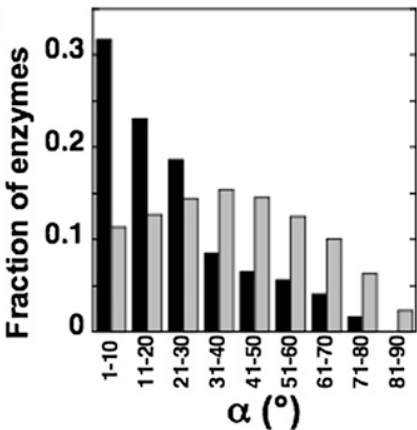

Fig. 7. Evaluating the angle between Asp and Glu general acids and bases and neighboring Phe residues. (A) The angle between the Asp or Glu residue and the Phe ring was defined by the closest carboxylate oxygen atom, the center point of the Phe ring, and the plane of the Phe ring. To match the angle convention used in ref. 9, an angle of $0^{\circ}$ corresponds to the carboxylate positioned near the ring edge of the Phe, and an angle of $90^{\circ}$ corresponds to the carboxylate positioned above the face of the Phe ring. $(B)$ Histogram of the analysis ( $n=253$ proteins) shows a preference for positioning the carboxylate oxyanion of Asp and Glu general acids and bases (black bars) near the edge of the ring that is greater than the distribution expected from available volume (gray bars; see Analysis of Angles Between Carboxylate General Acids and Bases and Neighboring Phe Residues for details). Values for the randomly distributed contacts are from Table S3. PDB files are listed in Table S4. A similar preference was observed for structural interactions (figure 4 in ref. 9).

and, thus, enhances catalysis via more efficient proton abstraction and ground-state destabilization (Fig. 9 and Table S1).

Although the actual evolution of KSI was certainly much more complex than this simplified scenario, this example illustrates why Phe residues may, in some instances, be favored over hydrogenbond networks in the positioning of anionic catalytic groups. Phe residues may have been present in more primitive enzymes with hydrophobic substrates that provide much of their rate enhancement via binding. The presence of Phe residues for binding may have then provided opportunities for the later coopting of these residues for the fine-tuning of the positioning of catalytic anions.
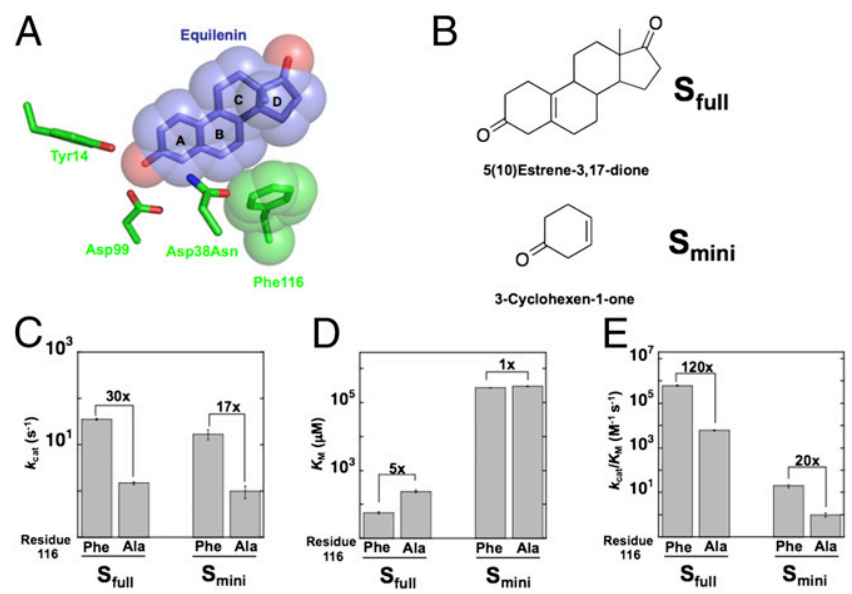

Fig. 8. Testing the role of Phe116 in remote substrate binding interactions. (A) Space-filling representation of the crystal structure of tKSI Asp38Asn bound with equilenin, a transition-state analog, shows the distal B-D steroid substrate rings packed against Phe116 (PDB ID code 1QJG; equilenin is colored violet, and Phe116 is colored green). (B) Structures of the multiple- and single-ring $\mathrm{KSI}$ substrates. Effects of the Phe116Ala mutation on $k_{\text {cat }}(C), K_{\mathrm{M}}$ $(D)$, and $k_{\text {cat }} / K_{\mathrm{M}}(E)$ for reaction using a multiple- and single-ring substrate. Values and errors are averages and SDs from three or more independent measurements and are from Table S1.

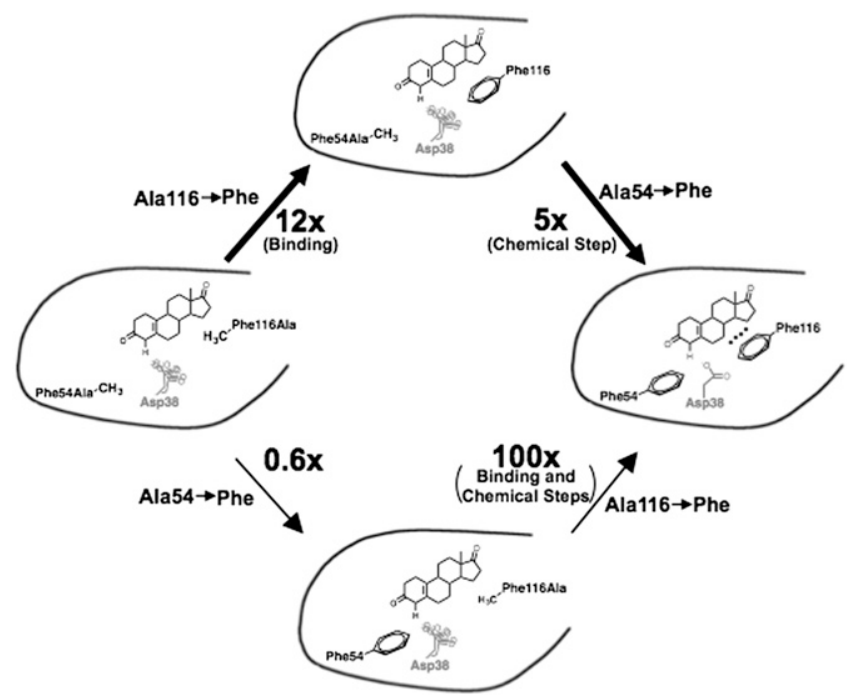

Fig. 9. Evolutionary scenario for anion-aromatic interactions positioning the general base in KSI. Relative $k_{\text {cat }} / K_{\mathrm{M}}$ values are given next to the arrows. The general base in the Phe54Ala/Phe116Ala double mutant is not positioned in the active site (light red, multiple conformations). Introduction of Phe54 alone does not lead to an increase in activity compared with double mutant (bottom path). However, in the top path (thicker arrows), introduction of Phe116 alone increases substrate binding (top species, black dots), and introduction of the second Phe leads to an effect on the chemical step by positioning the general base.

\section{Implications}

The cation-pi interaction is well recognized in biology, with hundreds of reports and numerous reviews in the literature of cation-pi interactions involved in ion channels, protein-ligand interactions, and cofactor binding (e.g., refs. 6, 7, 11, and 3640). Our results suggest a catalytic role for the related but lessdiscussed anion-aromatic interaction and provide strong support for a widespread role of anion-aromatic interactions in the positioning of anionic general bases in enzyme active sites. In addition, the ability of Phe residues to provide "double duty" in binding hydrophobic substrates and anion-aromatic interactions may have provided an impetus for the frequent use of Phe in the positioning of active-site general bases.

\section{Experimental Methods}

Materials. All reagents were commercially available and of the highest purity $(\geq 97 \%)$. All buffers were prepared with reagent-grade materials or better; $5(10)$ EST was from Steraloids; 3-cyclohexen-1-one was synthesized as described previously (35).

KSI Mutagenesis, Expression, and Purification. Quik-Change (Stratagene) sitedirected mutagenesis was used to introduce the mutations into th tKSI gene encoded on pKK22-3 plasmids or pET21c plasmids. Mutations were confirmed by sequencing miniprep DNA from DH5 $\alpha$ cells. Proteins were expressed and purified as described previously (41).

KSI Kinetics. Reactions with 5(10)EST ( $\left.S_{\text {full }}\right)$ and 3-cyclohexen-1-one $\left(S_{\text {mini }}\right)$ were monitored as described previously (35). Reactions were conducted at $25^{\circ} \mathrm{C}$ in $10 \mathrm{mM}$ potassium phosphate ( $\mathrm{pH}$ 7.2), $1 \mathrm{mM}$ sodium EDTA, $2 \mathrm{mM}$ DTT with $2 \%$ (vol/vol) DMSO added as a cosolvent for substrate solubility. The values of $k_{\mathrm{cat}}, K_{\mathrm{M}}$, and $k_{\mathrm{cat}} / K_{\mathrm{M}}$ were determined by fitting the initial rates as a function of substrate concentration (typically eight concentrations varied from 2 to $600 \mu \mathrm{M}$ and $0.02-800 \mathrm{mM}$ for reactions with $S_{\text {full }}$ and $S_{\text {mini, }}$ respectively) to the Michaelis-Menten equation. At least three determinations at differing enzyme concentrations (varying at least fourfold overall) were averaged.

KSI X-Ray Crystallography. Single-crystal diffraction data were collected at the Stanford Synchrotron Radiation Lightsource Beamline BL9-1 using a wavelength of $0.98 \AA$ (42). The reflections were indexed and integrated and with the programs XDS (43); the intensities were scaled, merged, and converted 
to amplitudes with SCALA and TRUNCATE (44). Molecular replacement was performed with AMoRe using the PDB ID code 3CPO structure, and refinement was performed with REFMAC5 $(45,46)$. Manual model building was carried out with COOT (47). Crystallographic refinement statistics are given in Supporting Information.

Analysis of Angles Between Carboxylate General Acids and Bases and Neighboring Phe Residues. A list of PDB files with active-site Asp and Glu residues was generated using the Catalytic Site Atlas (Version 2.2.12) (28, 29). Asp and Glu residues with a side-chain carboxylate oxygen atom within $6 \AA$ of the center of the Phe ring were identified using a Perl script and the list of PDB files. The Catalytic Site Atlas $(28,29)$, MACiE (30), BRENDA (31), and the primary literature were then used to determine whether the activesite Asp and Glu residues have been implicated as general acids and bases. Multiple structures of the same enzyme (e.g., varying bound ligands, crystallography conditions, resolution, or mutations) were identified manually and removed.

The angle of the interaction was determined by measuring the angle between the closest carboxylate oxygen atom, the center point of the Phe ring, and the plane of the Phe ring. In structures with multiple identical chains in the asymmetric unit, only the A chain was used in the analysis, and only the A conformer was used when multiple conformation existed in the structure.

The expected distribution of angles for Asp/Glu residues relative to Phe based on available volume was determined using the volume available to an

1. Fersht AR (1999) Structure and Mechanism in Protein Science (W. H. Freeman and Co., New York), 2nd Ed.

2. Jencks WP (1987) Catalysis in Chemistry and Enzymology (Dover, New York), 2nd Ed.

3. Carter P, Wells JA (1987) Engineering enzyme specificity by "substrate-assisted catalysis" Science 237(4813):394-399.

4. Carter P, Wells JA (1988) Dissecting the catalytic triad of a serine protease. Nature 332(6164):564-568.

5. Fersht AR, et al. (1985) Hydrogen bonding and biological specificity analysed by protein engineering. Nature 314(6008):235-238.

6. Dougherty DA (1996) Cation-pi interactions in chemistry and biology: A new view of benzene, Phe, Tyr, and Trp. Science 271(5246):163-168

7. Ma JC, Dougherty DA (1997) The Cationminus signpi Interaction. Chem Rev 97(5): 1303-1324.

8. Jackson MR, et al. (2007) A preference for edgewise interactions between aromatic rings and carboxylate anions: The biological relevance of anion-quadrupole interactions. J Phys Chem B 111(28):8242-8249.

9. Philip V, et al. (2011) A survey of aspartate-phenylalanine and glutamate-phenylalanine interactions in the protein data bank: Searching for anion- $\pi$ pairs. Biochemistry 50(14):2939-2950

10. Frontera A, Gamez P, Mascal M, Mooibroek TJ, Reedijk J (2011) Putting anion- $\pi$ in teractions into perspective. Angew Chem Int Ed Engl 50(41):9564-9583.

11. Salonen LM, Ellermann M, Diederich $F$ (2011) Aromatic rings in chemical and biological recognition: Energetics and structures. Angew Chem Int Ed Engl 50(21) 4808-4842.

12. Schottel BL, Chifotides HT, Dunbar KR (2008) Anion-pi interactions. Chem Soc Rev 37 (1):68-83.

13. Thomas KA, Smith GM, Thomas TB, Feldmann RJ (1982) Electronic distributions within protein phenylalanine aromatic rings are reflected by the three-dimensional oxygen atom environments. Proc Natl Acad Sci USA 79(16):4843-4847.

14. Holliday GL, Mitchell JB, Thornton JM (2009) Understanding the functional roles of amino acid residues in enzyme catalysis. $J$ Mol Biol 390(3):560-577.

15. Wu ZR, et al. (1997) Solution structure of 3-oxo-delta5-steroid isomerase. Science 276 (5311):415-418.

16. Cho HS, Choi G, Choi KY, Oh BH (1998) Crystal structure and enzyme mechanism of Delta 5-3-ketosteroid isomerase from Pseudomonas testosteroni. Biochemistry 37(23): 8325-8330.

17. Pollack RM, Bantia S, Bounds PL, Koffman BM (1986) pH dependence of the kinetic parameters for 3-oxo-delta 5-steroid isomerase. Substrate catalysis and inhibition by (3S)-spiro[5 alpha-androstane-3,2'-oxiran]-17-one. Biochemistry 25(8):1905-1911.

18. Kim DH, et al. (1999) Roles of active site aromatic residues in catalysis by ketosteroid isomerase from Pseudomonas putida biotype B. Biochemistry 38(42):13810-13819.

19. Alter GM, et al. (1990) Mutation of essential catalytic residues in pig citrate synthase. Biochemistry 29(33):7557-7563.

20. Zawrotny ME, Pollack RM (1994) Reaction energetics of a mutant 3-oxo-delta 5 steroid isomerase with an altered active site base (D38E). Biochemistry 33(46): 13896-13902.

21. Seebeck FP, Hilvert D (2005) Positional ordering of reacting groups contributes significantly to the efficiency of proton transfer at an antibody active site. J Am Chem Soc 127(4):1307-1312.

22. Raines RT, Sutton EL, Straus DR, Gilbert W, Knowles JR (1986) Reaction energetics of a mutant triosephosphate isomerase in which the active-site glutamate has been changed to aspartate. Biochemistry 25(22):7142-7154.

23. Vihinen $M$, Helin S, Mantsala $P$ (1991) Site-directed mutagenesis of putative activesite residues of Bacillus stearothermophilus $\alpha$-amylase. Mol Eng 1:267-273. oxygen atom within the cutoff distance of $6 \AA$ minus the distance excluded by the van der Waals spheres of benzene and the interacting oxygen. Volumes were calculated with the computer-aided design software SolidWorks (Dassault Systèmes) as follows. A model of benzene was constructed that included the van der Waals spheres for the benzene carbon and hydrogen atoms and a surrounding surface of a depth of $1.52 \AA$ to correspond to the van der Waals radius for oxygen. Conical sections were excised at $10^{\circ}$ increments, and volumes were calculated. The same procedure was followed for a sphere of $6 \AA$ to correspond to the cutoff distance from the center of the Phe ring. For each angular bin, these quantities were subtracted to give the volume available to the center of the interacting oxygen.

ACKNOWLEDGMENTS. We thank members of the laboratory of D.H. for comments on the manuscript. This work was funded by National Science Foundation Grant MCB-1121778 (to D.H.). J.P.S. and J.K.L. were supported in part by National Institutes of Health (NIH) Postdoctoral Fellowships. Portions of this research were carried out at the Stanford Synchrotron Radiation Laboratory, a national user facility operated by Stanford University on behalf of the US Department of Energy, Office of Basic Energy Sciences. The Stanford Synchrotron Radiation Lightsource Structural Molecular Biology Program is supported by the Department of Energy, Office of Biological and Environmental Research, and by the NIH, National Center for Research Resources (NCRR), Biomedical Technology Program, and the National Institute of General Medical Sciences. The project described was partially supported by Grant 5 P41 RR001209 from the NCRR, a component of the NIH.

24. Lawson SL, Wakarchuk WW, Withers SG (1996) Effects of both shortening and lengthening the active site nucleophile of Bacillus circulans xylanase on catalytic activity. Biochemistry 35(31):10110-10118.

25. Sippl MJ (1995) Knowledge-based potentials for proteins. Curr Opin Struct Bio/ 5(2): 229-235.

26. Poole AM, Ranganathan R (2006) Knowledge-based potentials in protein design. Curr Opin Struct Biol 16(4):508-513.

27. Fernandez-Ballester G, Serrano L (2006) Prediction of protein-protein interaction based on structure. Methods Mol Biol 340:207-234.

28. Porter CT, Bartlett GJ, Thornton JM (2004) The Catalytic Site Atlas: A resource of catalytic sites and residues identified in enzymes using structural data. Nucleic Acids Res 32D129-D133.

29. Bartlett GJ, Porter CT, Borkakoti N, Thornton JM (2002) Analysis of catalytic residues in enzyme active sites. J Mol Biol 324(1):105-121.

30. Holliday GL, et al. (2007) MACiE (Mechanism, Annotation and Classification in Enzymes): Novel tools for searching catalytic mechanisms. Nucleic Acids Res 35D515-D520.

31. Scheer M, et al. (2011) BRENDA, the enzyme information system in 2011. Nucleic Acids Res 39D670-D676.

32. Consortium TU; UniProt Consortium (2012) Reorganizing the protein space at the Universal Protein Resource (UniProt). Nucleic Acids Res 40(Database issue):D71-D75.

33. Kim SW, et al. (1997) High-resolution crystal structures of delta5-3-ketosteroid isomerase with and without a reaction intermediate analogue. Biochemistry 36(46): 14030-14036.

34. Ruben EA, et al. (2013) Ground state destabilization from a positioned general base in the ketosteroid isomerase active site. Biochemistry 52(6):1074-1081.

35. Schwans JP, Kraut DA, Herschlag D (2009) Determining the catalytic role of remote substrate binding interactions in ketosteroid isomerase. Proc Natl Acad Sci USA 106(34):14271-14275

36. Gallivan JP, Dougherty DA (1999) Cation-pi interactions in structural biology. Proc Natl Acad Sci USA 96(17):9459-9464.

37. Meyer EA, Castellano RK, Diederich F (2003) Interactions with aromatic rings in chemical and biological recognition. Angew Chem Int Ed Engl 42(11):1210-1250.

38. Dougherty DA (2008) Physical organic chemistry on the brain. J Org Chem 73(10): 3667-3673.

39. Zacharias N, Dougherty DA (2002) Cation-pi interactions in ligand recognition and catalysis. Trends Pharmaco/ Sci 23(6):281-287.

40. Scrutton NS, Raine AR (1996) Cation-pi bonding and amino-aromatic interactions in the biomolecular recognition of substituted ammonium ligands. Biochem J 319(Pt 1):1-8

41. Kraut DA, Sigala PA, Fenn TD, Herschlag D (2010) Dissecting the paradoxical effects of hydrogen bond mutations in the ketosteroid isomerase oxyanion hole. Proc Natl Acad Sci USA 107(5):1960-1965.

42. Soltis SM, et al. (2008) New paradigm for macromolecular crystallography experiments at SSRL: Automated crystal screening and remote data collection. Acta Crystallogr D Biol Crystallogr 64(Pt 12):1210-1221.

43. Kabsch W (2010) XDS. Acta Crystallogr D Biol Crystallogr 66(Pt 2):125-132.

44. Collaborative Computational Project, Number 4 (1994) The CCP4 suite: Programs for protein crystallography. Acta Crystallogr D Biol Crystallogr 50(Pt 5):760-763.

45. Murshudov GN, Vagin AA, Dodson EJ (1997) Refinement of macromolecular structures by the maximum-likelihood method. Acta Crystallogr D Biol Crystallogr 53(Pt 3): 240-255.

46. Krissinel EB, et al. (2004) The new CCP4 Coordinate Library as a toolkit for the design of coordinate-related applications in protein crystallography. Acta Crystallogr D Biol Crystallogr 60(Pt 12 Pt 1):2250-2255.

47. Emsley P, Cowtan K (2004) Coot: Model-building tools for molecular graphics. Acta Crystallogr D Biol Crystallogr 60(Pt 12 Pt 1):2126-2132. 